\title{
The exponential sum over squarefree integers
}

\author{
by \\ Jan-Christoph Schlage-Puchta (Freiburg)
}

Denote by $r_{\nu}(N)$ the number of representations of $N$ as the sum of $\nu$ squarefree numbers. In a series of papers Evelyn and Linfoot [3]-[8] proved that

$$
r_{\nu}(N)=\mathfrak{S}_{\nu}(N) N^{\nu-1}+\mathcal{O}\left(N^{\nu-1-\theta(\nu)+\varepsilon}\right),
$$

where

$$
\mathfrak{S}_{\nu}(N)=\frac{1}{(\nu-1) !}\left(\frac{6}{\pi^{2}}\right)^{\nu} \prod_{p^{2} \nmid N}\left(1-\frac{1}{\left(1-p^{2}\right)^{\nu}}\right) \prod_{p^{2} \mid N}\left(1-\frac{1}{\left(1-p^{2}\right)^{\nu-1}}\right),
$$

and

$$
\theta(2)=\theta(3)=\frac{1}{3}, \quad \theta(\nu)=\frac{1}{2}-\frac{1}{2 \nu} \quad(\nu \geq 4) .
$$

Mirsky [9] improved the error term for $\nu \geq 3$ to

$$
\theta(\nu)=\frac{1}{2}-\frac{1}{4 \nu-2} .
$$

Using a new approach to bound the minor arc integral developed by Brüdern, Granville, Perelli, Vaughan and Wooley [1], Brüdern and Perelli [2] showed that $\theta=1 / 2$ for all $\nu \geq 3$, and that any further improvement would imply a quasiriemannian hypothesis. Moreover, assuming the generalized riemannian hypothesis, they proved that $\theta(3)=3 / 4+1 / 14$ and $\theta(\nu)=3 / 4$ for all $\nu \geq 4$. These results are optimal apart from the summand $1 / 14$; in a personal communication Brüdern conjectured that $\theta(3)=3 / 4$ should hold true. It is the aim of this note to prove this conjecture.

Define $S(\alpha)=\sum_{n \leq N} \mu^{2}(n) e(\alpha n)$. For integers $N$ and $Q$ satisfying $1 \leq$ $Q<N^{1 / 2} / 2$, let $\mathfrak{M}(Q)$ be the union of all intervals $\left\{\alpha:|\alpha q-a| \leq Q N^{-1}\right\}$, where $q \leq Q$, and $(a, q)=1$. Set $\mathfrak{m}(Q)=\left[Q N^{-1}, 1-Q N^{-1}\right] \backslash \mathfrak{M}(Q)$. With this notation we will prove the following.

Theorem 1. We have $S(\alpha) \ll N^{1+\varepsilon} Q^{-1}$ for all $\alpha \in \mathfrak{m}(Q)$, provided that $Q \leq N^{1 / 2}$.

Key words and phrases: squarefree integers, exponential sum, circle method. 
Under the restriction $Q \leq N^{3 / 7}$, this was proven in [2, Theorem 4]. As already remarked in [2, Sec. 5], the weakening of the assumption on $Q$ implies the following.

Theorem 2. Assume the generalized riemannian hypothesis. Then

$$
r_{3}(N)=\mathfrak{S}(N) N^{2}+\mathcal{O}\left(N^{5 / 4+\varepsilon}\right) .
$$

By Dirichlet's theorem on diophantine approximation, for every $\alpha \in \mathfrak{m}(Q)$ there exist coprime integers $a, q$ with $q \leq N Q^{-1}$ such that $|q \alpha-a| \leq N^{-1} Q$. By the definition of $\mathfrak{m}(Q)$, we necessarily have $q>Q$. Hence, Theorem 1 is essentially equivalent to the following.

Theorem 3. Define $S(\alpha)$ as above, and let $q$ be an integer satisfying $|\alpha q-a| \leq q^{-1}$. Then

$$
|S(\alpha)| \ll N^{1+\varepsilon} q^{-1}+N^{\varepsilon} q .
$$

We approach Theorem 3 by the following lemma, which replaces Lemma 1 in [2].

Lemma 1. Let $\alpha \in(0,1)$ be a real number, and assume that $|q \alpha-a|$ $<1 / q$. Let $D$ be an integer, and denote by $W(D, z)$ the number of integers $d \leq D$ satisfying $\left\|d^{2} \alpha\right\| \leq z$. Then, for $D^{2}>\frac{1}{4} q$, we have

$$
W(D, z) \ll D^{2} q^{-1}+D^{1+\varepsilon} z^{1 / 2} .
$$

Proof. Decompose the interval $\left[1, D^{2}\right]$ into $K=\left[D^{2} q^{-1}\right]+1$ intervals of length $q$, where the last interval may be shorter. For $k \leq K$, let $a_{k}$ be the number of integers $d$ such that $\left\|d^{2} \alpha\right\| \leq z$ and $k q \leq d^{2}<(k+1) q$. Then $\sum_{k \leq K} a_{k}=W(D, z)$, and by the arithmetic-quadratic mean inequality, $\sum_{k \leq K} a_{k}^{2} \geq W(D, z)^{2} K^{-1}$. Denote by $\mathcal{D}$ the set of all pairs $\left(d_{1}, d_{2}\right)$ with $\left\|d_{i}^{2} \alpha\right\| \leq z$ and $1 \leq\left|d_{1}^{2}-d_{2}^{2}\right| \leq q$. Then either $W(D, z) \leq 2 K$, which is sufficiently small, or we can bound $|\mathcal{D}|$ from below via

$$
|\mathcal{D}| \geq \sum_{k}\left(\begin{array}{c}
a_{k} \\
2
\end{array}\right) \gg \sum_{k} a_{k}^{2}-\sum_{k} a_{k} \gg \sum_{k} a_{k}^{2} \gg W(D, z)^{2} K^{-1} .
$$

Denote by $\mathcal{N} \subseteq[1, q]$ the set of all values of $\left|d_{1}^{2}-d_{2}^{2}\right|$, where $d_{1}, d_{2}$ range over all pairs in $\mathcal{D}$. Then every pair in $\mathcal{D}$ gives rise to an element of $\mathcal{N}$, and the number of different pairs $d_{1}, d_{2}$ having the same difference $d_{1}^{2}-d_{2}^{2}=n$ is bounded above by the number of divisors of $n$, and therefore $\ll q^{\varepsilon}$. Hence, we deduce

$$
W(D, z)^{2} \ll|\mathcal{D}| K \ll|\mathcal{N}| K q^{\varepsilon} .
$$

On the other hand, for every $n \in \mathcal{N}$, we have $\|n \alpha\| \leq\left\|d_{1}^{2} \alpha\right\|+\left\|d_{2}^{2} \alpha\right\| \leq 2 z$, hence

$$
W(D, z)^{2} \ll D^{2} q^{\varepsilon-1}|\{n \leq q:\|\alpha n\| \leq 2 z\}| \ll D^{2} q^{\varepsilon-1}(q z+1) .
$$


From this, in the case $W(D, z)>2 K$ we obtain

$$
W(D, z) \ll D^{1+\varepsilon} z^{1 / 2}+D^{1+\varepsilon} q^{-1 / 2},
$$

which is again of the right size, since $D>\frac{1}{2} q^{1 / 2}$.

Proof of Theorem 3. Write

$$
\begin{aligned}
S(\alpha) & =\sum_{d \leq \sqrt{N}} \mu(d) \sum_{m \leq N d^{-2}} e\left(\alpha d^{2} m\right) \\
& \ll \log N \max _{1 \leq D \leq \sqrt{N} / 2} \sum_{D \leq d<2 D} \min \left(\frac{N}{D^{2}},\left\|\alpha d^{2}\right\|^{-1}\right) \\
& =\log N \max _{1 \leq D \leq \sqrt{N} / 2} \Upsilon(\alpha, D),
\end{aligned}
$$

say. To prove Theorem 3 , it suffices to show that $\Upsilon(\alpha, D) \ll N^{1+\varepsilon} Q^{-1}$ for all $D \leq \sqrt{N} / 2$. For $D>\frac{1}{4} q^{1 / 2}$, we have

$$
\begin{aligned}
\Upsilon(\alpha, D) & \ll \log N \max _{z>N / D^{2}} z^{-1} W(D, z) \\
& \ll \log N \max _{z>N / D^{2}}\left(z^{-1} D^{2} q^{-1}+D^{1+\varepsilon} z^{-1 / 2}\right) \ll N^{1+\varepsilon} q^{-1}+N^{1 / 2+\varepsilon} .
\end{aligned}
$$

For $D \leq \frac{1}{4} q^{1 / 2}$, we argue as in the proof of [2, Lemma 1$]$. We have

$$
\left|\alpha d^{2}-\frac{a d^{2}}{q}\right| \leq 4 D^{2}\left|\alpha-\frac{a}{q}\right| \leq 4 D^{2} q^{-2} \leq \frac{1}{4 q},
$$

and therefore

$$
|\Upsilon(\alpha, D)| \leq 2 \sum_{D \leq d<2 D}\left\|\frac{a d^{2}}{q}\right\| \ll q \log q \ll N^{\varepsilon} q .
$$

Taking these estimates together, we find that

$$
S(\alpha) \ll N^{1+\varepsilon} q^{-1}+N^{1 / 2+\varepsilon}+N^{\varepsilon} q,
$$

and the second term is always dominated by either the first or the last one, which implies our theorem.

\section{References}

[1] J. Brüdern, A. Granville, A. Perelli, R. C. Vaughan and T. D. Wooley, On the exponential sum over $k$-free numbers, Philos. Trans. Roy. Soc. London Ser. A 356 (1998), 739-761.

[2] J. Brüdern and A. Perelli, Exponential sums and additive problems involving squarefree numbers, Ann. Scuola Norm. Sup. Pisa Cl. Sci. (4) 28 (1999), 591-613.

[3] C. J. A. Evelyn and E. H. Linfoot, On a problem in the additive theory of numbers I, Math. Z. 30 (1929), 433-448.

[4] - - -, On a problem in the additive theory of numbers II, J. Reine Angew. Math. 164 (1931), 131-140. 
[5] C. J. A. Evelyn and E. H. Linfoot, On a problem in the additive theory of numbers III, Math. Z. 34 (1932), 637-644.

[6] - - - On a problem in the additive theory of numbers IV, Ann. of Math. 32 (1931), 261-270.

[7] - - - On a problem in the additive theory of numbers V, Quart. J. Math. Oxford Ser. 3 (1932), 152-160.

[8] —, - On a problem in the additive theory of numbers VI, ibid. 4 (1933), 309-314.

[9] L. Mirsky, On a theorem in the theory of numbers due to Evelyn and Linfoot, Proc. Cambridge Philos. Soc. 44 (1948), 305-312.

Mathematisches Institut

Universität Freiburg

Eckerstr. 1

79104 Freiburg, Germany

E-mail: jcp@mathematik.uni-freiburg.de 